\title{
Gamma heavy chain disease in a patient with rheumatoid arthritis - a laboratory evaluation
}

\author{
Wibke Johannis*, Jenny Blommer, Andreas R. Klatt, Joerg H. Renno, Klaus Wielckens \\ Institute for Clinical Chemistry, University Hospital of Cologne, Koeln, Germany \\ *Corresponding author: wibke.johannis@uk-koeln.de
}

\begin{abstract}
Introduction: Heavy chain diseases $(H C D)$ are neoplastic proliferations of $B$ cells which secrete truncated immunoglobulin heavy chains without associated light chains. Being rare and probably underdiagnosed diseases the aim of this report is to show an additional case of gamma heavy chain disease in a 48 year old female patient with rheumatoid arthritis focusing on the laboratory presentation.
\end{abstract}

Materials and methods: Laboratory work-up included agarose gel electrophoresis (AGE), capillary zone electrophoresis (CZE), immunofixation and nephelometrically determined immunoglobulin and immunoglobulin subclasses of the patient's serum. Urine samples were also subjected to immunofixation and to a SDS-PAGE with consecutive immunoblot.

Results: Nephelometrically measured elevated IgG concentrations were noted in combination with a decreased gamma globulin region and an increased beta globulin region on AGE. A definite monoclonal spike was not identified on AGE but at least suspected on CZE; finally serum and urine immunofixation demonstrated a monoclonal gamma heavy chain devoid of any corresponding light chains confirming the diagnosis of HCD. Analysis of the gamma heavy chain (HC) with means of SDS-PAGE revealed proteins of $40 \mathrm{kD}$ and $80 \mathrm{kD}$ most likely presenting a truncated HC in its monomeric and dimeric form and possibly leading to the failure of IgG-subclass typing with the applied lgG subclass antisera.

Conclusion: This case report illustrates a new case of gamma HCD demonstrating variable laboratory manifestations and therefore the need for heightened awareness concerning this disease when confronted with abnormal and discrepant protein profiles in routinely applied laboratory tests.

Key words: heavy chain disease; arthritis, rheumatoid; immunoglobulin gamma-chains; electrophoresis; electrophoresis, capillary

\section{Introduction}

Heavy chain diseases (HCD) are rare B-cell lymphoplasma-cell proliferative disorders characterized by production of truncated monoclonal immunoglobulin heavy chains without associated light chains (1). The incidence of gamma heavy chain diseases is higher than for alpha- but lower than for mu- heavy chain diseases. To our knowledge approximately 125 cases of gamma heavy chain diseases have been reported in the literature (2-7). The underlying disorders are variable: disseminated lymphoplasma cell proliferative disorders (marked by constitutional symptoms and lymphadenopathy) and localized lymphoplasma cell proliferative disorders (extramedullary or medullary) seem to be more common than autoimmune disorders (most frequently Sjögren's syndrome and rheumatoid arthritis) (4). In the cases with underlying rheumatoid arthritis cytokines have been postulated as a pathogenic link. Especially interleukin 1, interleukin 6 and tumor necrosis factor-a are involved in the development of monoclonal gammopathies, including gamma HCD, as well as in the proliferation and activation of lymphocytes seen in rheumatoid arthritis (8). Apart from the clinical diversity a wide spectrum of pathological findings in laboratory tests has been described. Serum immunofixation documenting a monoclonal heavy chain in the absence of an according light chain is an important laboratory finding in gamma HCD whereas monoclonal spikes in serum protein electrophoresis with differing mobility are often but not consistently found; a variety of results in quantitative lgG 
measurements is a further phenomenon $(3,4)$. Detectability of the gamma $\mathrm{HC}$ in urine with means of immunofixation has also been seen in a variable number of cases (4). We therefore want to present an additional case of gamma HCD in rheumatoid arthritis, a rare and assumedly underdiagnosed disease, focussing on its presentation in predominantly routinely applied laboratory tests.

\section{Materials and methods}

\section{Case history}

A 48 year old female patient with a history of seronegative rheumatoid arthritis was seen by her general practitioner because of increasing joint pain in both hands for a diagnostic work-up. At this point she was not on any disease modifying drugs after having stopped taking methotrexate three years earlier due to elevated liver enzymes. The patient denied fatigue and loss of appetite or weight. On physical examination no lymphadenopathy or enlargement of liver or spleen was remarked. She was referred to a hemato-oncologist following the diagnosis of HCD; a bone marrow biopsy was not performed as there was no clinical evidence of a disseminated or localized lymphoplasma cell proliferative disorder.

\section{Methods}

Routine laboratory biochemistry tests (serum creatinine, urea and total protein; urine protein, urine albumin, urine alpha 1 microglobulin) were run on a Roche Modular P automated clinical chemistry analyzer (Roche Diagnostics, Mannheim, Germany) with original Roche reagents. Agarose gel electrophoresis (AGE) of serum proteins was run on a HYDRASYS system (Sebia, Issy-les-Moulinaux, France) according to the manufacturer's instructions. Serum capillary zone electrophoresis (CZE) was performed with the automated capillary zone electrophoresis system Capillarys 2 by Sebia. Semiautomated serum and urine immunofixation (24-hour urine specimen) was performed on a Sebia HYDRASYS system with $\operatorname{lgM}, \lg A$, IgG, kappa and lambda light chain antisera by Sebia. Nephelometric quantification of immunoglobulins (reagents by Siemens, Marburg, Germany) and immunoglobulin subclasses and free light chains (reagents by The Binding Site, Birmingham, UK) was performed on a BNProSpec analyzer (Siemens, Marburg, Germany). SDS-PAGE of the patient's urine was performed on NuPAGE Novex Midi Gels (Invitrogen, Carlsbad, USA) under reducing and nonreducing conditions. The proteins were then transferred onto a polyvinylidene difluoride (PVDF) membrane (Invitrogen) and immunoblotted with peroxidise-conjugated rabbit anti-human lgG (DAKO, Glastrup, Denmark). A complete blood count was performed in an external laboratory on a Sysmex XE-5000 analyzer (Sysmex Corporation, Kobe, Japan).

\section{Results}

Hematology tests performed in an external laboratory showed a normal erythrocyte, leukocyte and thrombocyte count. Total serum protein and serum renal function parameters were unremarkable. AGE of the patient's serum solely revealed quantitative abnormalities (Figure 1) whereas CZE additionally demonstrated an M-protein defining spike for one of three independent interpreters (Figure 2). Serum and urine immunofixation demonstrated broad and heterogeneous monoclonal bands in the IgG lane (Figures 3 and 4). Total lgG and the IgG1 subclass in serum were elevated. Proteinuria showed a borderline value in the presence of an elevated urine IgG (all quantitative results summarized in table 1). All IgG subclass measurements in urine and in a 25 fold concentration of the urine showed results beneath the detection limit (approximate detection limits: lgG1: $131 \mathrm{mg} / \mathrm{L}$, IgG2: 153 mg/L, IgG3: 2.7 mg/L, IgG4: 1.9 mg/L). SDS-PAGE of the patient's urine with a consecutive immunoblot presented 2 bands (approximately 40 $\mathrm{kD}$ and $80 \mathrm{kD}$ ) (Figure 5).

\section{Discussion}

We primarily found a disturbed morphology of the protein profile on AGE presenting in form of an increased beta globulin fraction and hypogammaglobulinemia in our patient with gamma HCD. Agarose gel electrophoresis did not reveal a characteristic visible monoclonal spike in the beta region, as 
a)

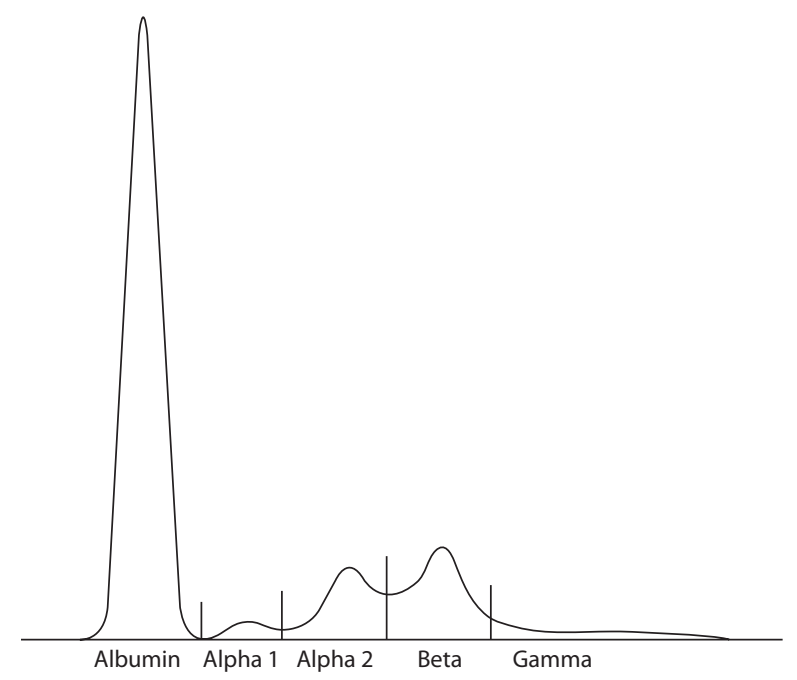

b)

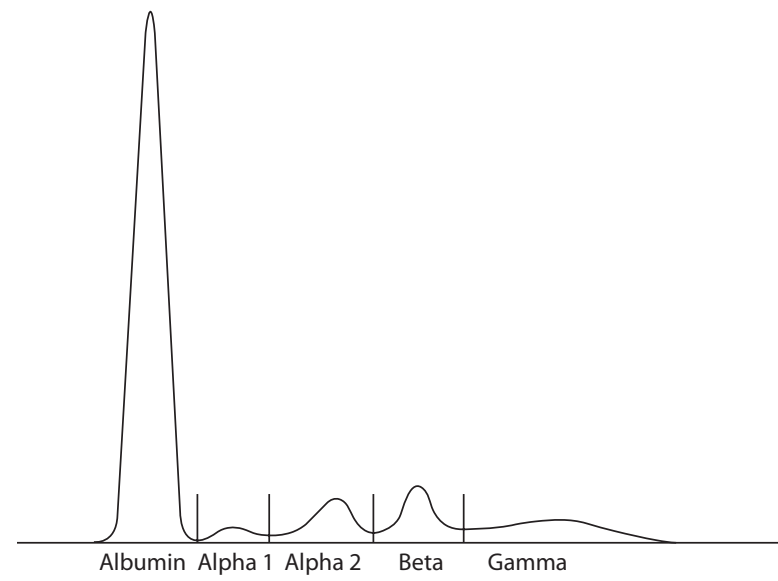

Figure 1. Agarose gel electrophoresis of a) the patient's serum proteins and $b$ ) a healthy control serum. An elevated beta and a decreased gamma region in the patient's serum can be noted in the absence of a monoclonal protein (M spike) as seen by three independent interpreters.

described for most patients with gamma HCD (4). Interestingly the capillary zone electrophoresis (CZE) did demonstrate a narrow based peak in the beta1 zone suggestive of a monoclonal protein component for at least one of three interpreters; a low gamma globulin concentration was also evident. Since assessing CZE is subjective, the additional elevated alpha1 and alpha2 fractions could be suggestive of an inflammatory response for two investigators. Nevertheless, this type of protein profile (on both AGE and CZE) would have lead to a subsequent serum immunofixation for all three investigators and two were possibly more cautious in their re- a)

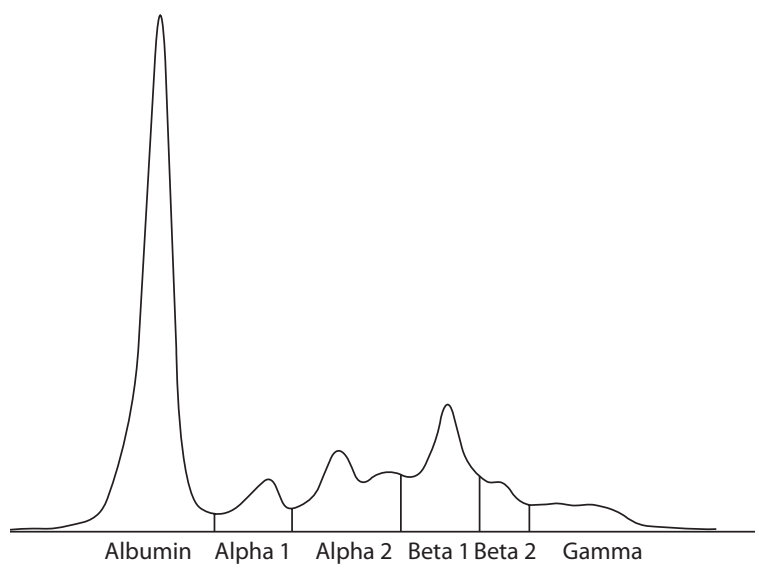

b)

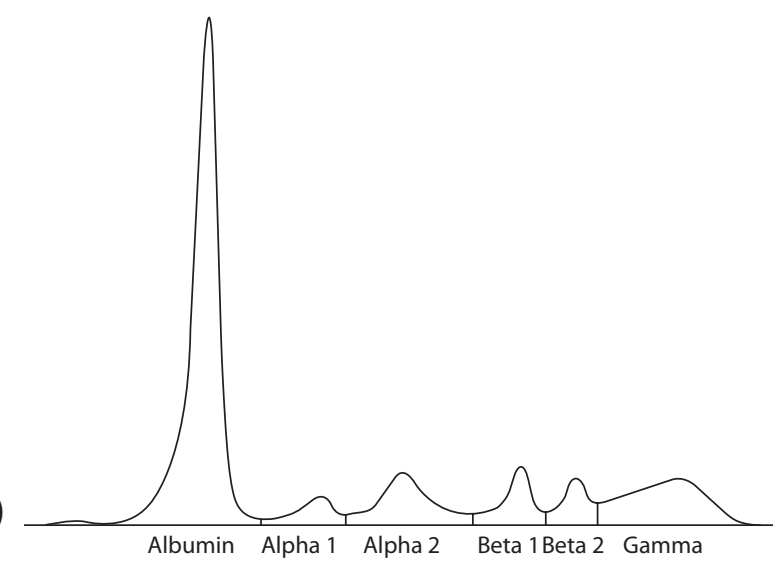

Figure 2. Capillary zone electrophoresis of a) the patient's serum proteins and $\mathrm{b}$ ) a healthy control serum. The alpha 1 , alpha 2 and beta 1 zones are elevated and the gamma zone is decreased in the patient's serum. A fairly narrow-based peak in the beta 1 zone in the patient's serum was seen as an M-protein defining spike by one of three independent interpreters.

porting of a monoclonal protein prior to seeing the results of serum immunofixation. Consequently as a singular applied diagnostic tool the CZE showed a higher reliability in finding the gamma $\mathrm{HC}$ in this particular case with the according interpreters compared to AGE but a conclusion on which method is better can't be made upon these results. There are multiple published studies comparing AGE and CZE for the detection of monoclonal proteins demonstrating following sensitivity rates: $91 \%(95 \% \mathrm{Cl} 86.2$ 95.6\%) vs. 92\% (95\% Cl 85.9-97.1\%) by McCudden et al., $99 \%$ vs. $97 \%$ by Bossuyt et al, $87 \%$ and $90 \%$ by Bakker et al. and 90\% (95\% Cl 81-98\%) vs. 81\% (95\% $\mathrm{Cl} 70-92 \%)$ by Yang et al. but the characterized specimens in these studies did not contain a HCD (9-12). 
Table 1. Laboratory results of quantitative tests. AGE: Agarose gel electrophoresis. CZE: Capillary zone electrophoresis.

\begin{tabular}{|c|c|c|}
\hline Laboratory test & Patient's results & Reference value \\
\hline Creatinine $(\mu \mathrm{mol} / \mathrm{L})$ & 40.5 & $<79.2$ \\
\hline Urea (mmol/L) & 2.9 & $<8.3$ \\
\hline Total protein (g/L) & 82 & $64-83$ \\
\hline Albumin fraction (\%) AGE & 63.5 & $60-71$ \\
\hline Alpha 1 fraction (\%) AGE & 2.4 & $1.4-2.9$ \\
\hline Alpha 2 fraction (\%) AGE & $\uparrow 12.1$ & $7-11$ \\
\hline Beta globulin fraction (\%) AGE & $\uparrow 16.4$ & $8-13$ \\
\hline Gamma globulin fraction (\%) AGE & $\downarrow 5.6$ & $9-16$ \\
\hline Albumin zone (\%) CZE & $\downarrow 51.3$ & $53.1-66.4$ \\
\hline Alpha 1 zone (\%) CZE & $\uparrow 5.9$ & $3.2-5.7$ \\
\hline Alpha 2 zone (\%) CZE & $\uparrow 16.3$ & $7.5-12.4$ \\
\hline Beta 1 zone (\%) CZE & $\uparrow 15.7$ & $5.2-8.1$ \\
\hline Beta 2 zone (\%) CZE & 4.7 & $3.4-6.5$ \\
\hline Gamma zone (\%) CZE & $\downarrow 6.1$ & $10.3-19.6$ \\
\hline Free kappa light chains (mg/L) & 6.96 & $3.3-19.4$ \\
\hline Free lambda light chains & 8.56 & $5.7-26.3$ \\
\hline Kappa / lambda quotient & 0.81 & $0.26-1.65$ \\
\hline $\lg G(g / L)$ & $\uparrow 25.8$ & $8-17$ \\
\hline $\operatorname{lgG1}(\mathrm{mg} / \mathrm{L})$ & $\uparrow 12400$ & $2800-8000$ \\
\hline $\operatorname{lgG} 2(\mathrm{mg} / \mathrm{L})$ & $\downarrow 1080$ & $1150-5700$ \\
\hline $\operatorname{lgG} 3(\mathrm{mg} / \mathrm{L})$ & 493 & $240-1250$ \\
\hline $\operatorname{lgG} 4(\mathrm{mg} / \mathrm{L})$ & $\downarrow 9$ & $52-1250$ \\
\hline $\lg M(g / L)$ & 0.48 & $0.4-2.3$ \\
\hline $\lg A(g / L)$ & 1.87 & $0.7-4.0$ \\
\hline Total urine protein $(\mathrm{g} / \mathrm{L})$ & $\uparrow 0.15$ & $<0.15$ \\
\hline Urine albumin (mg/L) & 9 & $<20$ \\
\hline Urine IgG (mg/L) & $\uparrow 226$ & $<10$ \\
\hline Urine alpha 1 microglobulin (mg/L) & 1.3 & $<12$ \\
\hline Urine free kappa light chains (mg/L) & 6.14 & $1.35-24.19$ \\
\hline Urine free lambda light chains (mg/L) & $<0.259$ & $0.24-6.66$ \\
\hline
\end{tabular}

Yang et al. report that in their study both methods "missed" samples with monoclonal IgA which often migrates in the beta region similar to our described gamma HC protein (12). Poisson et al. demonstrated an overall excellent sensitivity of $>90 \%$ for identification of monoclonal gammopathies with two different CZE systems using serum immunofixation as a gold standard but it should be acknowledged that 2 out of 5 samples with an IgA gammopathy were not found with one of these systems (13). Subopti- mal detection performances are therefore possibly due to the particular migration pattern even though Luraschi et al. reported a case where gamma HCD was detected in the course of routine serum assay by CZE through a peak in the beta region (14). The confirmation and identification of the monoclonal protein was performed with means of serum immunofixation revealing the presence of a monoclonal gamma band with no corresponding light chain; a finding consistent with the diagnosis of gamma 
a)

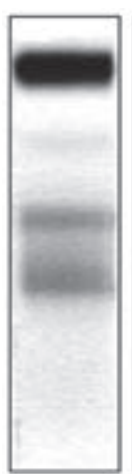

ELP

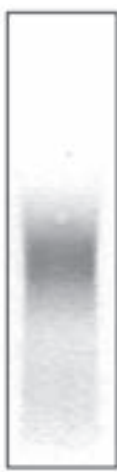

$\lg G$

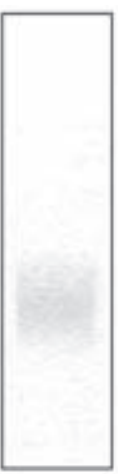

$\lg A$

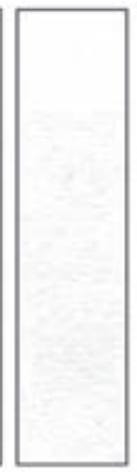

$\lg M$

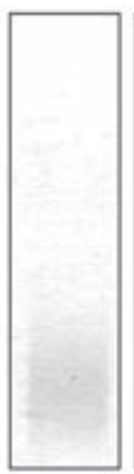

Kappa Lambda

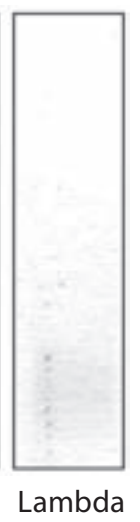

b)

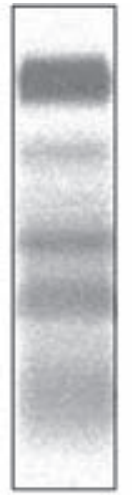

ELP

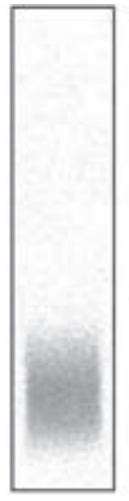

$\lg G$

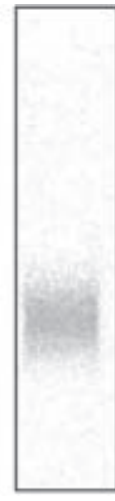

$\lg A$

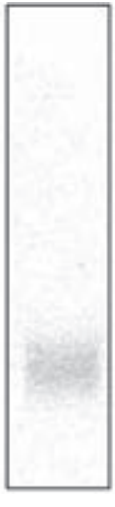

$\lg M$

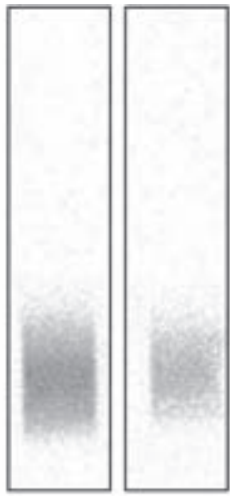

Kappa Lambda
Figure 3. Immunofixation of a) the patient's serum and b) a healthy control. The patient's immunofixation demonstrates a broad monoclonal IgG band with beta mobility when comparing to the electrophoresis lane without corresponding light chain. The healthy control serum in comparison shows polyclonal IgG in the gamma region in the absence of monoclonal bands.

ELP - serum protein electrophoresis; Kappa - Kappa light chains; Lambda - Lambda light chains.

HCD. The apparently same monoclonal component could be found in urine immunofixation and correspondingly lgG concentration was found to be elevated in the absence of albumin and alpha1 microglobulin. The detection of IgG without further proteinuria or signs of renal disease implies the presence of an abnormal lgG molecule. This was verified with means of SDS-PAGE and consecutive immunoblot demonstrating proteins with a molecular weight of approximately $40 \mathrm{kD}$ and $80 \mathrm{kD}$ reacting with anti-human IgG. A normal gamma heavy chain has a molecular weight of $51 \mathrm{kD}$; we therefore postulated the presence of a monomeric and dimeric form of truncated gamma heavy chain. Structural

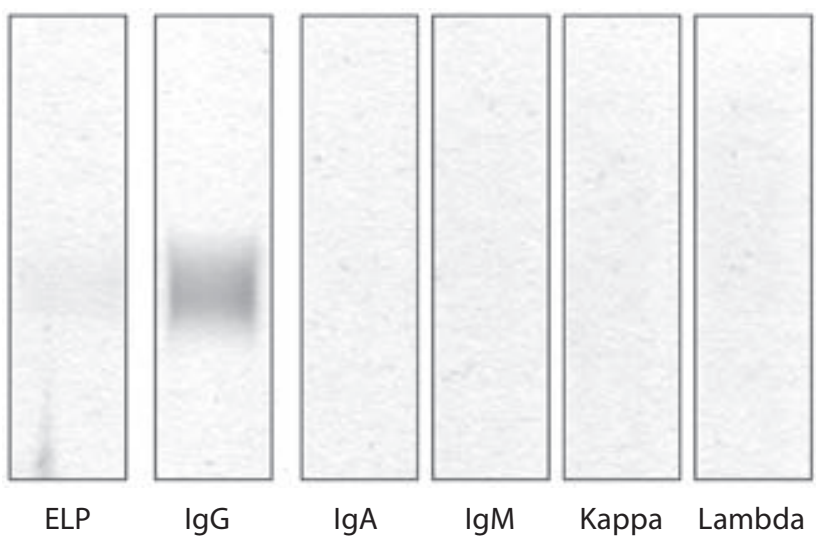

FIGURE 4. Immunofixation of the patient's urine proteins demonstrating a broad monoclonal lgG band without corresponding light chain. Other proteins were not detected in the electrophoresis lane. A healthy control would not show any bands. ELP - Urine protein electrophoresis; Kappa - Kappa light chains; Lambda - Lambda light chains.

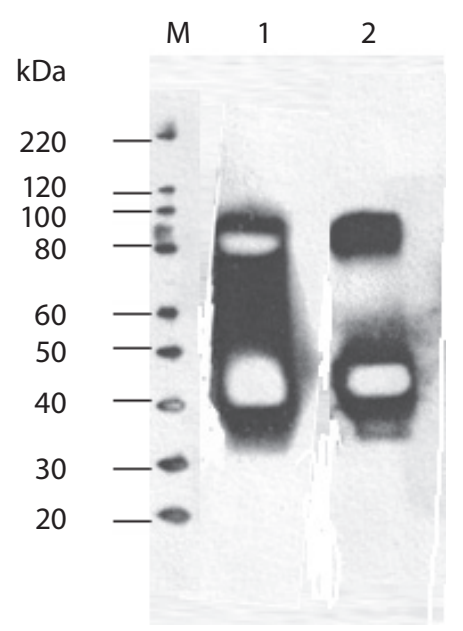

Figure 5. Western blotting of the patient's urine in the nonreduced (lane 1) and in the reduced form with beta-mercaptoethanol (lane 2). Under both conditions anti-human IgG reacted predominantly with an approximately $40 \mathrm{kD}$ protein band and additionally with an approximately $80 \mathrm{kD}$ band (signal weaker in the reduced land).

M - Marker.

protein abnormalities in form of deletions in the variable region or the constant domain in gamma HCD have been described leading to smaller (50-75\% of the normal gamma chain) gamma heavy chains which are partially prone to the formation of dimeric units; a partial reduction of the $80 \mathrm{kD}$ band supports the presence of gamma HCD dimers in our patient. The performed reduction with beta-mercaptoetha- 
nol was incomplete; the reason for this phenomenon remains ultimately unclear; the presence of a non disulfide bridge covalent link as seen for example in crosslinks in connective tissues is a possible but fairly bold explanation $(3,15)$. The immunofixation of serum and urine (lower detection limit for monoclonal lgG being $2.5 \mu \mathrm{g}$ as declared by the manufacturer) detected only one single broad band possibly due to a higher analytical sensitivity of the western blot (detecting less than $1 \mathrm{pg}$ of protein) in the setting of an altogether weaker $80 \mathrm{kD}$ band compared to the $40 \mathrm{kD}$ band (16). Another explanation is the different separation principle between both techniques, both relying on differences in protein size but immunofixation additionally on differences in protein charge. Serum IgG subclass typing demonstrated an elevated lgG1 concentration. IgG3 concentration was within normal limits and IgG2 and IgG4 were found to be decreased; the subclass sum $(13.9 \mathrm{~g} / \mathrm{L})$ was in discrepancy with total lgG levels $(25.8 \mathrm{~g} / \mathrm{L})$, the latter showing a markedly higher concentration. One explanation for the decreased lgG subclass values could have been an analyte excess overwhelming the binding capacity of the capture antibody (known as the hook effect), a problem commonly described in nephelometric assays but this was excluded with means of serum dilution (17). Immunoassays furthermore show an analytical variability as a result of differing detection antibody specificities (18). The phenomenon of discrepant values for total lgG and sum of lgG subclasses has

\section{References}

1. Franklin EC, Lowenstein J, Bigelow B, Meltzer M. Heavy chain disease - a new disorder of serum $\gamma$-globulins: report of the first case. Am J Med 1964;37:332-50.

2. Fernand JP, Brouet JC, Danon F, Seligmann M. Gamma heavy chain "disease": Heterogeneity of the clinicopathological features. Report of 16 cases and review of the literature. Medicine 1989;68:321-35.

3. Fernand JP, Brouet JC. Heavy-chain diseases. Hematol Oncol Clin North Am 1999;13:1281-94.

4. Wahner-Roedler DL, Witzig TE, Loehrer LL, Kyle RA. Gamma heavy chain disease. Review of 23 cases. Medicine 2003;82:236-50.

5. Munshi NC, Digumarthy S, Rahemtullah A. Case 13-2008: A 46-year-old man with rheumatoid arthritis and lymphadenopathy. N Engl J Med 2008;358:1838-48. been previously illustrated by other groups and might be explained by the loss of an antigenic domain normally recognized by the lgG subclass antisera due to truncation of the heavy chain $(6,19)$. We had primarily thought the elevated lgG1 subclass identifies the gamma HC subclass but we failed to subtype the detected IgG in (concentrated) urine supporting our assumption that the heavy chain did not react with our lgG1-4 antisera and the elevated IgG1 concentration in serum did not necessarily stand in connection with the gamma heavy chain. Alternatively the failure in detection of lgG subclasses in urine can possibly be explained by the applied test's detection limits but we excluded this option to a wide extent by demonstrating IgG subclass measurability in urine samples of patients with nephropathy displaying comparable total or even lower lgG concentrations. In summary we present a new case of gamma heavy chain disease demonstrating multiple laboratory findings in mostly routinely applied tests hoping to increase awareness for this disease when confronted with according results.

\section{Acknowledgements}

The authors want to kindly thank Dr. N. Karakaya for providing the necessary clinical information.

\section{Potential conflict of interest}

None declared.
6. Busser B, Millet S, Bulabois CE, Faure P, Renversez JC. Unusual increased beta-globulins in an elderly patient. Clin Chem 2011;57: 948-52.

7. Morita K, Kawamoto H, Takada H, Nakamura S, Ishii $K$ Okamoto $Y$. Unusual gamma heavy chain disease protein in a patient with splenic marginal-zone lymphoma. Ann Clin Biochem 2006;43:161-4.

8. Husby G. Is there a pathogenic link between gamma heavy chain disease and chronic arthritis? Curr Opin Rheumatol 2000;12:65-70.

9. McCudden CR, Mathews SP, Hainsworth SA, Chapman JF, Hammett-Stabler CA, Willis MS, Grenache DG. Performance comparison of capillary and agarose Gel electrophoresis for the identification and characterization of monoclonal immunoglobulins. Am J Clin Pathol 2008;129:451-8. 
10. Bossuyt X, Lissoir B, Marien G, Maisin D, Vunckx J, Blanckaert N, Wallemacq P. Automated serum protein electrophoresis by Capillarys. Clin Chem Lab Med 2003;41:704-10.

11. Bakker AJ, Elderman-van der Werf, van Abbema T. Detection and quantification of M-proteinemia: comparison of various methods for serum protein electrophoresis. Clin Chem Lab Med 2012;50:77-80.

12. Yang Z, Harrison $K$, Park YA, Chaffin CH, Thigpen B, Easley $P L$, et al. Performance of the Sebia CAPILLARYS 2 for detection and Immunotyping of serum monoclonal paraproteins. Am J Clin Pathol 2007;128:293-9.

13. Poisson J, Fedoriw Y, Henderson MPA, Hainsworth S, Tucker $K$, Uddin Z, McCudden CR. Performance evaluation of the Helena V8 capillary electrophoresis system. Clin Biochem 2012. doi:10.1016/j.clinbiochem.2012.03.018.

14. Luraschi P, Infusino I, Zorzoli I, Merlini G, Fundaro C, Franzini C. Heavy chain disease can be detected by Capillarys zone electrophoresis. Clin Chem 2005;51:247-9.
15. Wahner-Roedler DL, Kyle RA. Heavy chain diseases. Best Pract Res Clin Haematol 2002; 18:729-46.

16. Crisp SJ, Dunn MJ. Detection of proteins on protein blots using chemiluminescent systems. Meth $\mathrm{Mol}$ Biol 1994;32:233-7.

17. Dodig S. Interferences in quantitative immunochemical methods. Biochem Med 2009; 19:50-62.

18. Salamunic I. Laboratory diagnosis of autoimmune diseases - new technologies, old dilemmas. Biochem Med 2010;20:45-56.

19. Lee MT, Parwani A, Humphrey R, Hamilton RG, Myers DI, Detrick B. Gamma heavy chain disease in a patient with diabetes and chronic renal insufficiency: diagnostic assessment of the heavy chain fragment. J Clin Lab Anal 2008;22:146-50. 